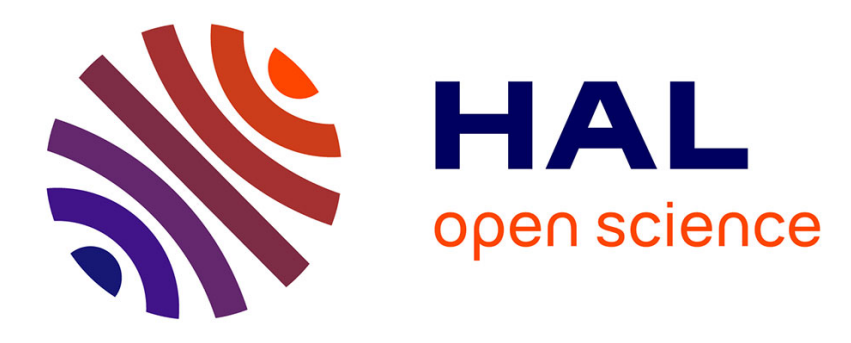

\title{
Bulk and interface scaling properties of the chiral clock model
}

J Yeomans, Bernard Derrida

\section{To cite this version:}

J Yeomans, Bernard Derrida. Bulk and interface scaling properties of the chiral clock model. Journal of Physics A : Mathematical and General, 1985, 18 (12), pp.2343-2355. 10.1088/0305-4470/18/12/031 . hal-03285916

\section{HAL Id: hal-03285916 https://hal.science/hal-03285916}

Submitted on 19 Jul 2021

HAL is a multi-disciplinary open access archive for the deposit and dissemination of scientific research documents, whether they are published or not. The documents may come from teaching and research institutions in France or abroad, or from public or private research centers.
L'archive ouverte pluridisciplinaire HAL, est destinée au dépôt et à la diffusion de documents scientifiques de niveau recherche, publiés ou non, émanant des établissements d'enseignement et de recherche français ou étrangers, des laboratoires publics ou privés. 


\title{
Bulk and interface scaling properties of the chiral clock model
}

\author{
Julia Yeomans $\nmid$ and Bernard Derrida $\ddagger$ \\ † Department of Theoretical Physics, 1 Keble Road, Oxford OX1 3NP, UK \\ $\ddagger$ Service de Physique Théorique, CEN Saclay, 91191 Gif-sur-Yvette, France
}

Received 12 November 1984, in final form 11 February 1985

\begin{abstract}
We use a finite-size scaling calculation to study the phase diagram of the two-dimensional, three-state, chiral clock model. Bulk properties, the ferromagnetic transition temperature and associated exponents are calculated from the scaling of the susceptibility and interface behaviour, the position of the interface wetting line and its exponents, from considering the net adsorption. We also present preliminary results on interface properties which are sensitive to the transition between the paramagnetic and incommensurate phases.
\end{abstract}

\section{Introduction}

The phase diagram of the two-dimensional chiral clock model (Huse 1981, Ostlund 1981) has proved complicated and controversial. The model exhibits many interesting features, a floating phase, chiral exponents, a Lifshitz point and an interfacial wetting transition even at zero temperature (see, e.g., Huse et al 1983, Duxbury et al 1984 and references therein).

In this paper we study the properties of strips to further elucidate the thermodynamic behaviour of the model. The work of Duxbury et al (1984) is extended by calculating the susceptibility, $\chi$, to obtain the phase boundary of the ferromagnetic phase and a combination of the bulk exponents. We then study the interface properties of the model to extract the position of the wetting transition and the associated exponents (Huse et al 1983). The results are compared to those obtained for a solid-on-solid model (Selke and Pesch 1982). Lastly we investigate several quantities which are sensitive to the appearance of the floating phase.

The two-dimensional chiral clock model (Ostlund 1981, Huse 1981) is described by the Hamiltonian

$$
\mathscr{H}=-J \sum_{\langle i j\rangle}^{\|} \cos \frac{2 \pi}{3}\left(n_{i}-n_{j}+\Delta\right)-J \sum_{\langle i j\rangle}^{\perp} \cos \frac{2 \pi}{3}\left(n_{i}-n_{j}\right)
$$

where a variable $n_{i}=0,1,2$ is associated with each site $i$ of a square lattice and the sums are taken over nearest neighbours in the axial $(\|)$ and perpendicular $(\perp)$ directions respectively. The phase diagram of the model is drawn schematically in figure 1 for $0<\Delta<\frac{1}{2}$. (The phase diagram for all other values of $\Delta$ may be obtained using symmetry arguments.) Three phases are stable: the ferromagnetic phase, $F$, the paramagnetic phase, $\mathrm{P}$, and the floating or incommensurate phase, I, which is characterised by 


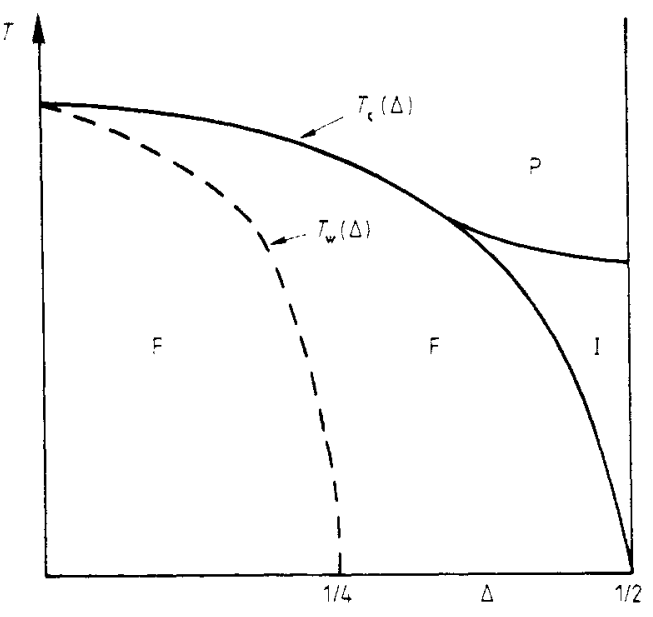

Figure 1. Schematic phase diagram of the two-dimensional chiral clock model. The broken curve corresponds to the interface wetting transition.

modulated order with a wavevector, $q$, which varies continuously with $T$ and $\Delta$. There is considerable controversy over the position of the Lifshitz point. Selke and Yeomans (1982) and Howes (1982) claim that the phase diagram is as drawn in figure 1 with a Lifshitz point at a finite value of $\Delta$. However Haldane et al (1983), Schultz (1983) and von Gehlen and Rittenberg (1983) argue that the floating phase extends to a Lifshitz point at $\Delta=0$.

Huse et al (1983) have recently pointed out that the chiral clock model has interesting interface properties. Consider an interface perpendicular to the axial direction. Because of the chiral nature of the interaction the free energy of an interface between spins taking values $n_{\mathrm{L}}, n_{\mathrm{R}}$ will depend on $\left(n_{\mathrm{R}}-n_{\mathrm{L}}\right)$ (modulo 3 ). Let us call a $+(-$ ) interface one for which $\left(n_{R}-n_{L}\right)$ (modulo 3 ) is 1 (2). For $\Delta=0$ the free energies of + and - interfaces, $F_{+}$and $F_{-}$respectively, are equal. However as $\Delta$ is increased $F_{+}$ decreases relative to $F_{-}$. When $2 F_{+}=F_{-}$it will be favourable for any - interface to split into two + interfaces and hence become wet.

We call the line where this happens $T_{\mathrm{w}}(\Delta)$; a consideration of ground-state energies shows that, for $\Delta=\frac{1}{4}, T_{\mathrm{w}}=0$. Huse et al (1983) have argued that $T_{\mathrm{w}}(\Delta)$ should increase with decreasing $\Delta$ and join the ferromagnetic-paramagnetic phase boundary at $\Delta=0$. For values of $T>T_{\mathrm{w}}(\Delta)$, they suggest that the decomposition of the - interfaces will lead to a change in the nature of the fluctuations and hence that the ferromagneticparamagnetic phase boundary could be in a new chiral universality class different from that of the three-state Potts model. Huse et al (1983) argued that the crossover exponent is positive and chirality is indeed relevant. Analytic but heuristic arguments given by den Nijs (1984) support this. However, no numerical evidence for any new exponent on this phase boundary has been obtained so far (Selke and Yeomans 1982, Duxbury et al 1984).

In this paper we calculate the exact thermodynamic properties of infinite strips of width $N$ (non-boundary) sites and use finite size scaling (Barber 1983) to extract the properties of the two-dimensional system. In $\$ 2$ we consider the susceptibility of strips with the infinite direction taken along the axial direction and with periodic boundary conditions perpendicular to the strip. This enables us to estimate the ferromagnetic 
phase boundary and the exponent $\gamma / \nu_{\perp}$. We then repeat the same calculation for a different orientation of the strip, the axial direction now being perpendicular to the infinite direction of the strip.

In $\S 3$ we present results for the excess number of the non-boundary state generated by the interface. Looking for the line where this quantity scales with the strip width, gives estimates for the wetting line, $T_{\mathrm{w}}(\Delta)$, and the corresponding critical exponents. The extent to which the results can be reproduced by a solid-on-solid model of the interface is discussed in $\S 4$. Finally, in $\S 5$ we present some preliminary results which indicate a phase transition at finite temperature for $\Delta=\frac{1}{2}$ which corresponds to the appearance of the incommensurate phase.

\section{Scaling of the susceptibility}

The free energy per spin of a strip of width $N, F^{N}$, is obtained as usual (Camp and Fisher 1972) from the largest eigenvalue, $\lambda^{N}$, of the transfer matrix

$$
F^{N}=-(T / N) \log \left(\lambda^{N}\right) \text {. }
$$

If $H$ is the field which couples to state 0 we may define a susceptibility by

$$
\chi^{N}=\partial^{2} F^{N} / \partial H^{2} \text {. }
$$

We first choose the chiral direction to lie parallel to the infinite direction of the strip and take periodic boundary conditions across the strip. Hence no quantisation of the wavevector is imposed by the finite strip width.

The susceptibility is expected to scale in the vicinity of the ferromagnetic phase boundary, $T_{\mathrm{c}}(\Delta)$, as

$$
\chi^{N}(T) \sim N^{\gamma / \nu}-f\left(N^{1 / \nu}+\left(T-T_{c}\right)\right)
$$

where $\nu_{\perp}$ is the exponent of the correlation length perpendicular to the axial direction. In general this is expected to differ from the exponent in the axial direction, $\nu_{\|}$, because of the chiral nature of the transition. To extract the critical temperature and the critical exponent we compare three strips of sizes $N+1, N$ and $N-1$ (Kinzel and Yeomans 1981) and calculate the curve $T_{c}^{N}(\Delta)$ which solves the equality

$$
X^{N}\left(T_{\mathrm{c}}^{N}\right)=X^{N-1}\left(T_{\mathrm{c}}^{N}\right)
$$

where

$$
X^{N}(T)=\log \left(X^{N+1} / X^{N}\right) / \log ((N+1) / N) .
$$

We then expect

$$
\lim _{N \rightarrow \infty} X^{N}\left(T_{\mathrm{c}}^{N}\right)=\frac{\gamma}{\nu_{\perp}}
$$

and

$$
\lim _{N \rightarrow \infty} T_{\mathrm{c}}^{N}(\Delta)=T_{\mathrm{c}}(\Delta)
$$

The estimates of the phase boundary of the ferromagnetic phase shown in figure 2 were obtained from these calculations. The results converge well as can be checked for $\Delta=0$ where the exact value of $T_{c}$ is known to be 1.4925 . They are in agreement 


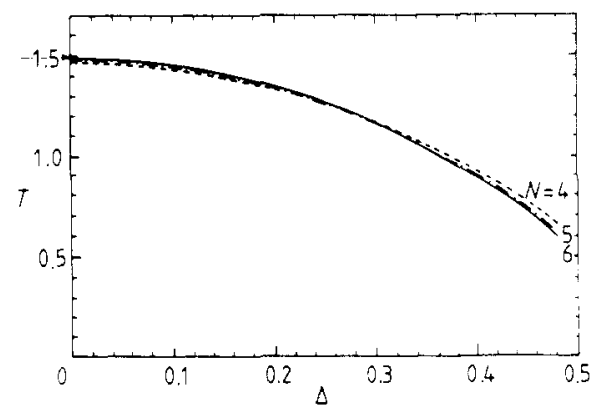

Figure 2. Estimates of $T_{c}(\Delta)$ from scaling the susceptibilities of three strips of widths $N-1$, $N$ and $N+1$. The chiral direction is parallel to the infinite direction of the strip. The exact value of $T_{\mathrm{c}}(\Delta=0)$ is indicated by an arrow.

with previous strip calculations (Duxbury et al 1984) in which different thermodynamic quantities, the correlation length and the wavevector of the modulated order, were scaled and with Monte Carlo results (Selke and Yeomans 1982).

The values of the ratio $\gamma / \nu_{\perp}$ obtained from (7) are shown in figure 3. For $\Delta=0$, where the exact value of $\gamma / \nu_{\perp}=\frac{26}{15}=1.733$ is known (Wu 1982) they seem to converge well.

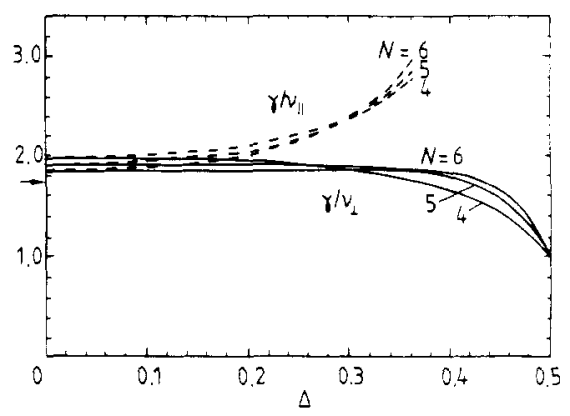

Figure 3. Exponent estimates from scaling the susceptibility. The full curves show $\gamma / \nu_{+}$, obtained with the chiral direction along the strip; the broken curves show $\gamma / \nu_{\|}$, obtained with the chiral direction perpendicular to the strip. The exact value for $\Delta=0$ is indicated by an arrow.

The results suggest that the ratio $\gamma / \nu_{\perp}$ is independent of $\Delta$ except for $\Delta=0.5$ where it takes the value of 1 . Perhaps it is not surprising that we see no crossover to chiral exponents for the ratio $\gamma / \nu_{\perp}$ as all previous numerical work suggests that even if the exponents are changed by the relevant operator it is only by a small amount (Selke and Yeomans 1982, Howes 1982, Duxbury et al 1984).

It is interesting to compare calculations identical except that the long direction of the strip is taken perpendicular to the chiral direction. Results for $T_{c}(\Delta)$ are very similar for $\Delta \leqslant 0.35$ and cannot be distinguished on the scale of figure 2 . However for $\Delta \geqslant 0.35$ the iteration routine which found the largest eigenvalue of the transfer matrix converged much more slowly and the Newton's method that we used to find the value of $T_{\mathrm{c}}$ which solves (5) failed to find a fixed point. 
Along the transition line, the ratio $\gamma / \nu_{\|}$seems to increase continuously with $\Delta$ (see the broken curves on figure 3) suggesting (as $\gamma / \nu_{\perp}$ is constant) a variation of the ratio $\nu_{\perp} / \nu_{\|}$. For $\Delta \geqslant 0.3$, however, the estimates of the ratio $\gamma / \nu_{\|}$increase with $N$ and do not seem to converge in the limit $N \rightarrow \infty$. The reason for the poor convergence is probably that the axial direction is perpendicular to the strip. This forces the system to have quantised values of the wavevector, $q$, whereas in the infinite system $q$ would vary continuously with $T$ and $\Delta$. However it is possible that the bad convergence of $\gamma / \nu_{\|}$and the fact that we do not find any fixed point for $\Delta \geqslant 0.35$ are reflecting the presence of the Lifshitz point.

\section{The interface wetting transition}

In this section we describe the application of finite strip calculations to the interface properties of the chiral clock model. The interface is introduced by taking the strip to lie perpendicular to the axial direction and fixing its infinite boundaries in states 0 and 2 as shown in figure 4 for $N=4$. In general interface properties are extracted by comparing the results to those on a strip with both boundaries fixed in state 0 which should describe a system with no interface in the thermodynamic limit. Because the finite dimension of the strip must lie along the axial direction to introduce the interface correctly the results should be viewed with circumspection as $\Delta$ increases because the system will be unable to attain its preferred wavevector. However, it seems reasonable that the wetting line, which lies within the ferromagnetic phase, should not be affected.

An interesting quantity is the net adsorption of the non-boundary state, 1 , defined by

$$
W_{1}^{N}(T, \Delta)=\mathcal{N}_{1}^{(0,2)}(T, \Delta)-\mathcal{N}_{1}^{(0,0)}(T, \Delta)
$$

where $\mathcal{N}_{\gamma}^{(\alpha, \beta)}$ is the average number of spins per row in state $\gamma$ with boundary conditions $(\alpha, \beta)$ (Selke and Pesch 1982). We expect $W_{1}^{N}(T, \Delta)$ to have a finite limit when $N \rightarrow \infty$

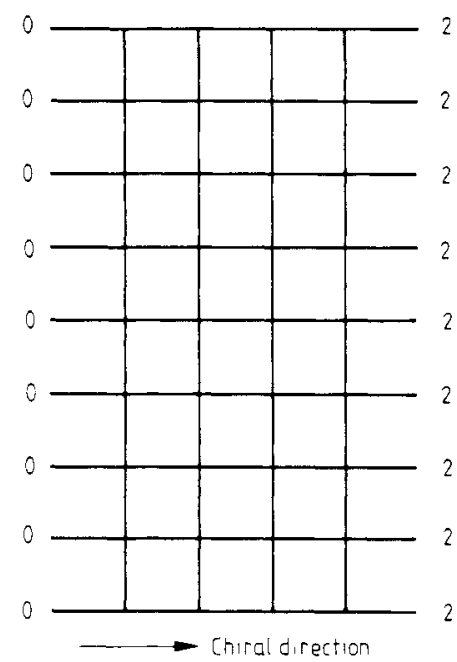

Figure 4. A strip of width $N=4$ showing the boundary conditions used to introduce an interface. 
for $T<T_{\mathrm{w}}(\Delta)$, when the interface is not wet, to scale as $N$ in the wet phase $T_{\mathrm{w}}(\Delta)<T<$ $T_{c}(\Delta)$, because the $0-1$ and 1-2 interfaces are independent of each other in the thermodynamic limit, and tend to a finite limit in the paramagnetic phase.

$W_{1}^{N}(T, \Delta)$ is plotted as a function of temperature for $N=2$ to 6 and various values of $\Delta$ in figure 5. For $\Delta=0$ the curves show a maximum at a value of the temperature which appears to converge to $T_{\mathrm{c}}=1.4925$, the exact result for the three-state Potts model. For $T>T_{\mathrm{c}}, W_{1}^{N}(T, \Delta)$ decreases with increasing $N$ and will become zero in the thermodynamic limit; for $T<T_{\mathrm{c}}, W_{1}^{N}(T, \Delta)$ converges to a finite value (with increasing $N$ ).

For $\Delta=0.15$ similar behaviour is observed. The maximum occurs at a lower temperature reflecting the decrease in $T_{\mathrm{c}}$ and $T_{\mathrm{w}}$. The wet phase is expected (Huse et al 1983) to extend over a small but finite range of temperature for this value of $\Delta$ but our results are not sensitive enough to confirm this. In general $W_{1}^{N}(T, \Delta)$ increases with increasing $\Delta$ as it becomes more favourable to include non-boundary states at the interface.
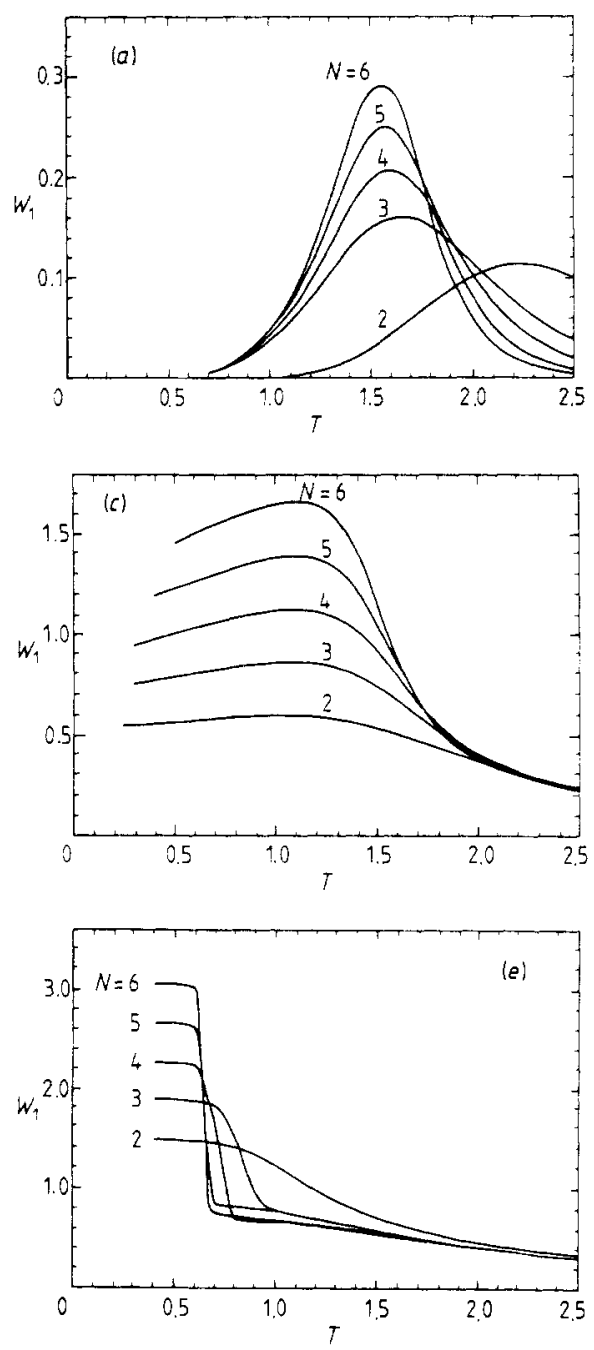
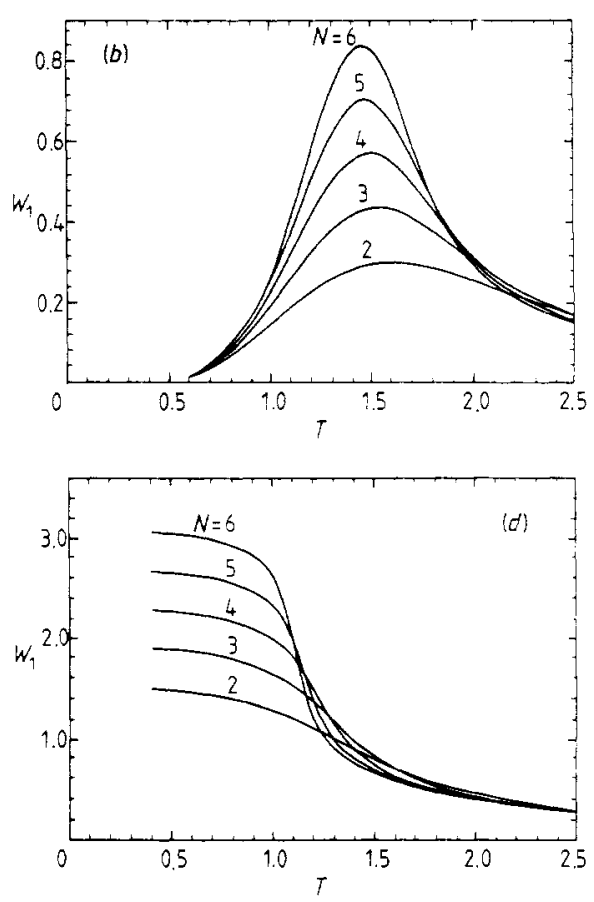

Figure 5. Net adsorption of the non-boundary state, $W_{1}^{N}(T, \Delta)$ as a function of temperature for $(a) \Delta=0$, (b) $\Delta=0.15$, (c) $\Delta=0.25$, (d) $\Delta=0.4$, (e) $\Delta=0.48$. (Note that the vertical scale changes with $\Delta$.) 
Note that for temperatures $T>T_{c}, W_{1}^{N}(T, \Delta)$ is virtually independent of $N$ showing that the quantity converges to a non-zero limit at all temperatures. To understand this effect we adopt the convention that the label in (1) increases from left to right (consistent with the boundary conditions in figure 4). Hence for $\Delta \neq 0$ the spin configuration $\ldots 012012 \ldots$ is preferable to . $210210 \ldots$ and, if the left-hand wall is in a state $n_{i}$, a larger fraction of spins in its vicinity will be in a state $\left(n_{i}+1\right)$ (modulo 3 ) than $\left(n_{i}-1\right)$ (modulo 3$)$. Let these fractions be $p_{+}(T, \Delta)$ and $p_{-}(T, \Delta)$ respectively. For a right-hand wall in state $n_{i}$ the fractions of neighbouring spins in states $\left(n_{i}+1\right)$ (modulo 3 ) and $\left(n_{i}-1\right)$ (modulo 3$)$ are $p_{-}$and $p_{+}$respectively. Hence the contribution to $\mathcal{N}_{1}^{(0,0)}$ due to this wall effect is $p_{+}+p_{-}$and the contribution to $\mathcal{N}_{1}^{(0,2)}$ is $2 p_{+}$. This gives a contribution to $W_{1}$ of $p_{+}-p_{-}$. which is zero only for infinite temperature or $\Delta=0$.

The main difference in the curves for larger values of $\Delta(0.25,0.4,0.48)$ is that $W_{1}^{N}(T, \Delta)$ tends to a finite limit at low temperatures showing, as expected, that the interface is wet throughout the ferromagnetic phase. It is expected that, for large $N, W_{1}^{N}(T, \Delta)$ will scale as $N$ throughout the wet phase. Although one sees clearly in figures $5(c)-(e)$ that $W_{1}$ increases linearly with $N$ it seems that the data would be best fitted by taking $W_{1} \sim A(N+C)$. One origin of the shift, $C$, is the wall effect described above but other effects may contribute such as the ambiguity in the definition of $N$ (see figure 4 where, for a strip of width $N$, an interface may lie in $N+1$ positions) and the width of each of the two interfaces due to their fluctuations which prevents them lying too close to each other or to the boundaries. For $\Delta=0.48, W_{1}$ appears to undergo a first-order transition at a temperature $\sim T_{c} \sim 0.6$ but this may well be a consequence of the quantisation of the wavevector. Results for $\Delta=0.5$ are postponed to $\$ 5$.

We now consider what information can be extracted from scaling properties of the wetting curves. Define the quantity

$$
Y^{N}(T)=\ln \left(W_{1}^{N+1} / W_{1}^{N}\right) / \ln ((N+1) / N) \text {. }
$$

In the limit of large $N$ the curves of $Y^{N}(T)$ as a function of temperature should be coincident throughout the wet region reflecting the scaling of the net adsorption with $N$. This cannot occur for strips of finite size and we find that for $\Delta \geqslant 0.05$ the scaling condition

$$
Y^{N}(T)=Y^{N-1}(T)
$$

holds at two values of the temperature which appear to converge to the boundaries of the wet region $T_{\mathrm{c}}(\Delta)$ and $T_{\mathrm{w}}(\Delta)$ in the thermodynamic limit.

Using this assumption we obtain the phase diagram shown in figure 6 . The upper curve gives a reasonable approximation to $T_{c}(\Delta)$ but lies rather higher than the values obtained from the susceptibility presumably due to finite-size effects and false quantisation of the wavevector. The lower curve describes the wetting transition $T_{w}(\Delta)$. It rises sharply from $\Delta=0.25$ and approaches $T_{c}$ rather more slowly than predicted by Monte Carlo calculations (Selke 1984). The two curves approach $\Delta=0$ with a high degree of tangency as expected (Huse et al 1983) and for $\Delta \simeq 0.05$ the two fixed points merge and (11) has no solution. It is not surprising that calculations on such small strips fail to reproduce accurately the point at which the two phase boundaries meet.

Consider now the critical exponents associated with the net adsorption. We expect that for large enough $N$

$$
W_{1}^{N}(T) \sim N^{z_{w}} f\left(N^{1 / \nu_{w}}\left(T-T_{w}\right)\right)
$$




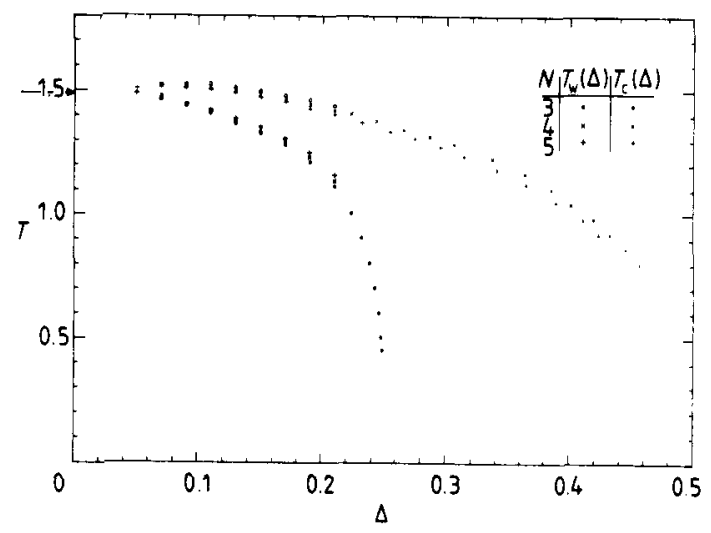

Figure 6. Estimates for the critical, $T_{c}(\Delta)$, and wetting, $T_{w}(\Delta)$, curves of the chiral clock model obtained from the scaling of the net adsorption, $W_{1}^{N}(T, \Delta)$. The exact value of $T_{\mathrm{c}}(\Delta=0)$ is indicated by an arrow.

$$
W_{1}^{N}(T) \sim N^{z_{c}} f\left(N^{1 / \nu_{\mathrm{c}}}\left(T-T_{\mathrm{c}}\right)\right)
$$

in the vicinity of $T_{\mathrm{w}}(\Delta)$ and $T_{\mathrm{c}}(\Delta)$ respectively. Values of $z_{\mathrm{w}}$ and $z_{\mathrm{c}}$ are plotted in figure 7. For $\Delta=0$ one expects $z_{\mathrm{w}}=z_{\mathrm{c}}=0.87$ (Selke and Huse 1983). For $0<\Delta \leqslant 0.2$, $z_{\mathrm{w}}$ and $z_{\mathrm{c}}$ remain equal and rather constant at a value $\approx 0.96$. However for increasing $N$ their values increase and we cannot rule out a slow convergence to one in the thermodynamic limit. For $\Delta \geqslant 0.2, z_{\mathrm{w}}$ crosses over to a new value and consideration of strips of different width suggests that the crossover occurs only for $\Delta=0.25$ in the thermodynamic limit. $z_{\mathrm{c}}$ also decreases quickly but converges badly allowing no interpretation of the results in this region.

One difficulty in working with the quantity $W_{1}^{N}(T, \Delta)$ defined in (9) is that, as discussed earlier in this section, it includes a contribution from the surfaces of the strip as well as from the interface. As a result $W_{1}^{N}$ has a finite limit when $N \rightarrow \infty$ in the high-temperature phase. In order to suppress this wall effect one can consider the

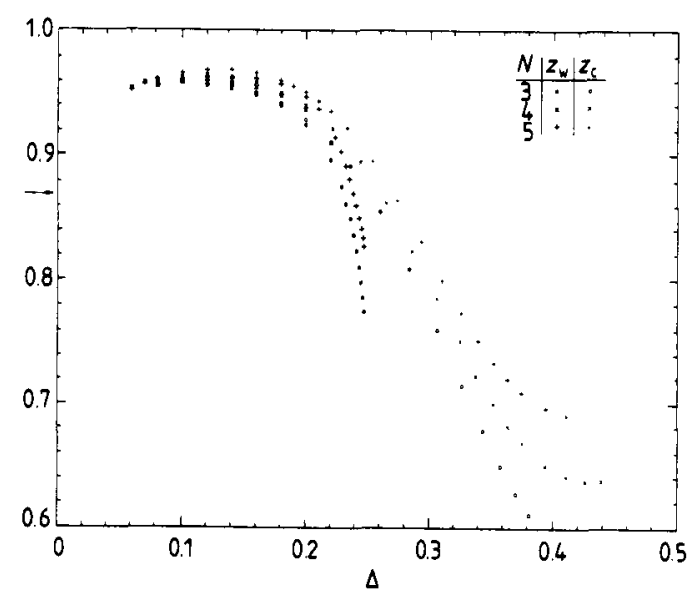

Figure 7. Wetting exponents, $z_{w}$ and $z_{c}$, of the chiral clock model obtained from the scaling of the net adsorption, $W_{1}^{N}(T, \Delta)$. The exact value for $\Delta=0$ is indicated by an arrow. 
combination $W_{1}+W_{2}$ where

$$
W_{2}^{N}(T, \Delta)=\mathcal{r}_{2}^{(0,1)}(T, \Delta)-\mathfrak{v}_{2}^{(0,0)}(T, \Delta)
$$

because, as one can convince oneself using an argument like that presented above, the surface term does not contribute to this quantity and it vanishes as $N \rightarrow \infty$ in the paramagnetic phase. We found that the results obtained using the new scaling variable were almost identical to those which resulted from scaling $W_{1}$.

\section{A solid-on-solid model}

It is interesting to compare the results of $\$ 3$ to those obtained for a solid-on-solid model in which only fluctuations of the interface and not of the bulk are taken into account. Huse et al (1983) argue that they expect the wetting line of the chiral clock model to be in the same universality class as that of a solid-on-solid model.

The solid-on-solid model we consider (Selke and Pesch 1982) is shown in figure 8 for $N=4$. An interface is introduced by fixing opposite boundaries of an infinite strip of width $N$ sites in states 0 and 2 . The interface is allowed to be wet and therefore two variables in each row are needed to describe it. $h_{i}$ and $l_{i}$ label the positions of the $0-1$ and $1-2$ interfaces respectively and obey the constraints $0 \leqslant h_{i} \leqslant l_{i} \leqslant N$. The Hamiltonian of the model is taken to be

$$
\begin{aligned}
\mathscr{H}=\sum_{i} \frac{3 J}{2}\left[\mid h_{i}-\right. & h_{i+1}|+| l_{i}-l_{i+1} \mid-\left(h_{i}-l_{i+1}\right) \theta\left(h_{i}-l_{i+1}\right) \\
& \left.-\left(h_{i+1}-l_{i}\right) \theta\left(h_{i+1}-l_{i}\right)\right]+\sum_{i}\left(2 J \cos \frac{2 \pi \Delta}{3}-2 J \cos \frac{2 \pi}{3}(1-\Delta)\right. \\
& +\delta_{h_{i}, l_{i}}\left(2 J \cos \frac{2 \pi}{3}(1-\Delta)-J \cos \frac{2 \pi \Delta}{3}-J \cos \frac{2 \pi}{3}(2-\Delta)\right)
\end{aligned}
$$

where $\theta$ denotes the step function and the energy is measured relative to the ferromagnetic state.

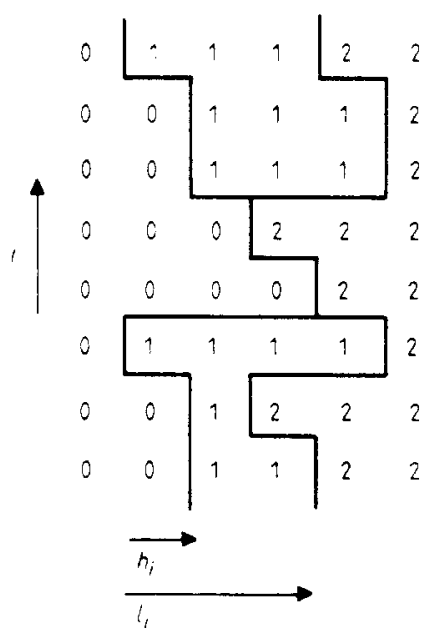

Figure 8. A solid-on-solid version of the chiral clock model. $N=4$ for the strip shown. 
As in the previous section we consider the net adsorption of the non-boundary state 1 defined by

$$
W_{1}^{N}(T, \Delta)=\mathcal{N}_{1}^{(0,2)}(T, \Delta)
$$

where, for this model, it is unnecessary to subtract a bulk contribution. Our aim is to study the transition temperature $T_{\mathrm{w}}^{\mathrm{SOS}}(\Delta)$ and the exponent $z_{\mathrm{w}}^{\mathrm{sos}}$ defined in (12) and compare them to the results for the chiral clock model. For the solid-on-solid model the size of the transfer matrix varies much less rapidly with $N$ and it is therefore easy to obtain results for larger values of $N$ than in $\S 3$.

The results are shown in figures 9 and 10. As expected they are identical to those obtained for the chiral clock model for low temperature $(T \leqslant 1.0)$ as overhangs of the

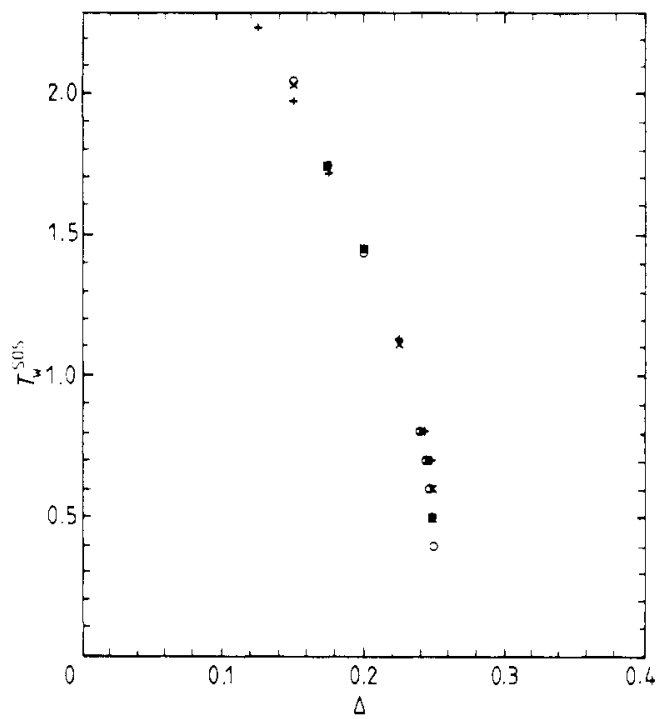

Figure 9. Wetting curve of the solid-on-solid model, $T_{w}^{\text {sos }}(\Delta)$, obtained from scaling the net adsorption (O,N=5; $\times, N=7 ;+, N=9$ ).

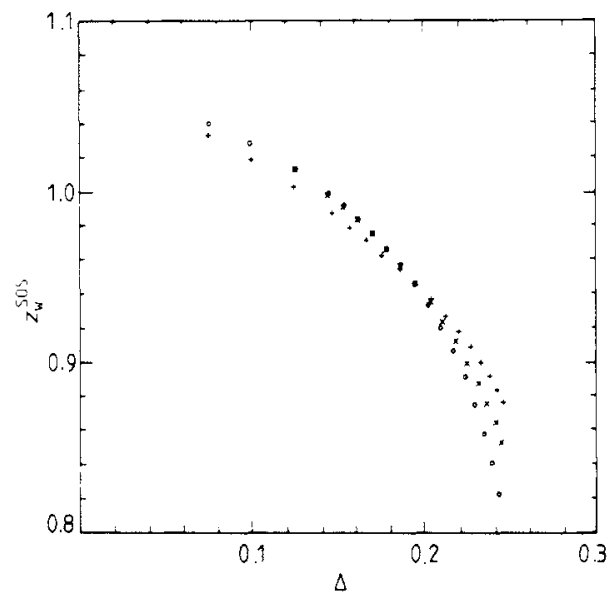

Figure 10. Critical exponent of the solid-on-solid model, $z_{w}^{\text {sos }}$, obtained from scaling the net adsorption $(O, N=5 ; \times, N=7 ;+, N=9$ ). 
interface boundaries and bubbles of a different state within the interface, which are ignored within the solid-on-solid approximation, are unimportant excitations. For larger values of the temperature the wetting curve rises sharply with decreasing $\Delta$. This reflects the lack of a divergent bulk correlation length in the solid-on-solid model.

The critical exponent shows some variation with $\Delta$ but consideration of strips of different sizes is consistent with $z_{\mathrm{w}}^{\mathrm{sOS}}$ taking the value 1 along the wetting curve and crossing over to a different value at the special point $(\Delta=0.25, T=0)$. Our results show that the behaviour of the exponent $z_{\mathrm{w}}$ is somewhat different in the chiral clock model and in its solid-on-solid version but they are not inconsistent with $z_{\mathrm{w}}=1$ in both cases.

\section{Preliminary results for $\Delta=0.5$}

For $\Delta=0.5$ the chiral clock model is expected to exhibit a transition between the paramagnetic phase and an incommensurate phase with algebraic decay of correlations (Ostlund 1981). It has proved very difficult to obtain accurate numerical results for the position of this phase boundary. The peak in the specific heat is thought to lie above the true transition (Selke and Yeomans 1982) and finite-size scaling results, which considered the correlation length (Duxbury et al 1984) converged very slowly. In this section we present preliminary results which show that interface properties are sensitive to the existence of the paramagnetic-incommensurate transition in the hope that this will lead to more quarititative work in the future.

Figure 11 shows the net absorption, $W_{1}^{N}(T, \Delta)$ defined in $(9)$, as a function of temperature for different values of $N$. For $T \leqslant 0.9$ the convergence becomes very slow and irregular. This at least indicates an increase in the correlation length along the axial direction. The irregularities occur because the system is sometimes prevented from locking into its preferred wavevector, which for this value of $\Delta$, is $q=2 \pi / 6$ (Ostlund 1981).

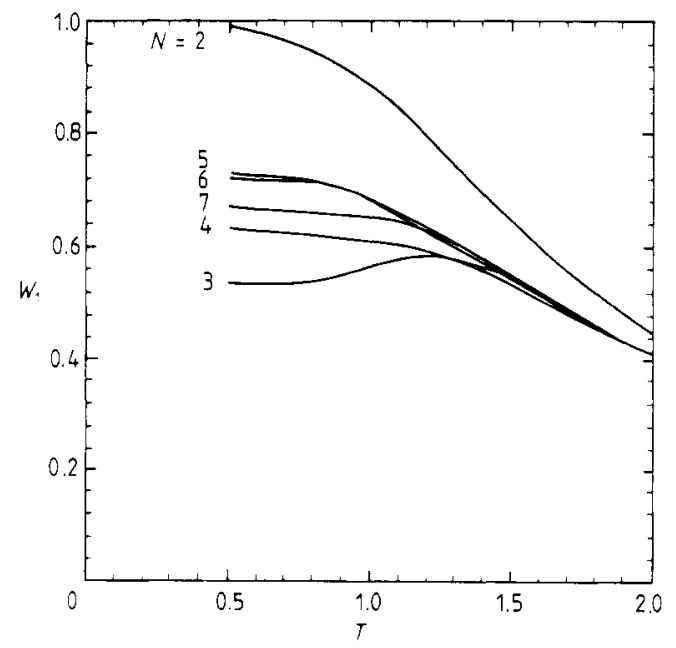

Figure 11. Net adsorption of the non-boundary state, $W_{1}^{N}(T, \Delta)$ as a function of temperature for $\Delta=0.5$. The chiral direction is perpendicular to the strip. 
Figure 12 also shows the net absorption but now with the axial direction taken to lie along the strip so that the interface lies parallel to the chiral axis. The net absorption has a very pronounced maximum which varies in a regular way with $N$ at a temperature $T \simeq 0.7$. This is probably indicating the transition to the incommensurate phase. It is interesting to note that in the low-temperature phase, the $N$ dependence looks very similar to that seen in the wet phase: it seems that for $\Delta=0.5$ even an interface parallel to the axial direction is wet.

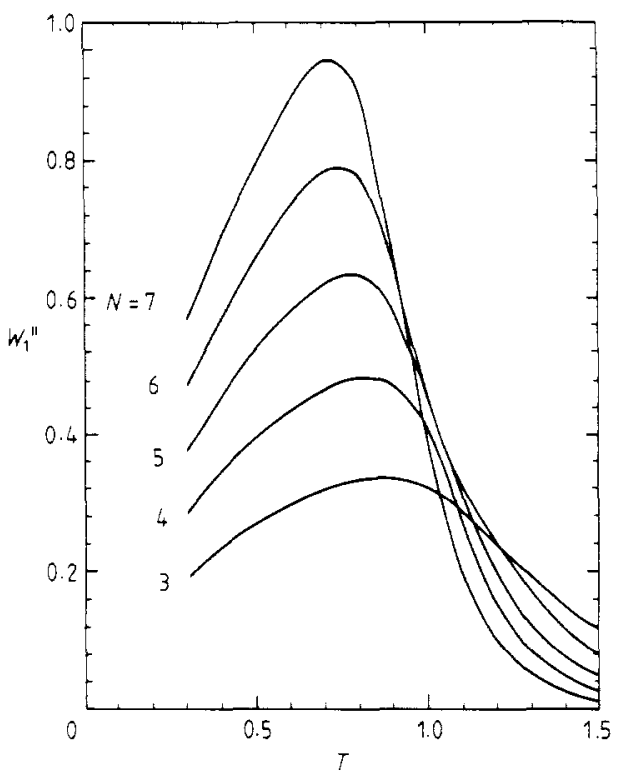

Figure 12. As for figure 11 but with the chiral direction parallel to the strip.

Lastly we considered systems with the axial direction still parallel to the strip but with periodic boundary conditions. The system was forced to have an interface along the strip by replacing the contribution to the Hamiltonian of one row of bonds perpendicular to the strip $J \Sigma_{\langle i j\rangle}^{\perp} \cos (2 \pi / 3)\left(n_{i}-n_{j}\right)$ by $J \Sigma_{\langle i j\rangle}^{\perp} \cos (2 \pi / 3)\left(n_{i}-n_{j}+1\right)$. The free energy of this interface, $\sigma^{N}$, as a function of the strip width is shown in figure 13. Clearly at high temperatures, $\sigma^{N}$ converges quickly to zero as $N$ increases.

At lower temperatures, $T \leqslant 1.0, \sigma^{N}$ still decreases with $N$ but much more slowly. We tried to see whether the results were consistent with $\sigma^{N}$ going to zero exponentially with $N$ as in the high-temperature phase or like a power law but the sizes we could obtain, $N \leqslant 7$, proved too small to distinguish the two possibilities. All the curves indicate that at $T \sim 0.9$ there is a phase transition confirming the results obtained by Houlrik et al (1983) from a Monte Carlo renormalisation group study. However, it is not obvious to us how to scale these curves in a way that will accurately determine the critical point or its associated exponents.

To conclude, we have studied the phase diagram of the three-state chiral clock model in two dimensions using finite-size scaling techniques. Phase boundaries and critical exponents have been calculated for bulk and interface transitions by scaling the bulk susceptibility and the net absorption respectively. We have shown that certain 


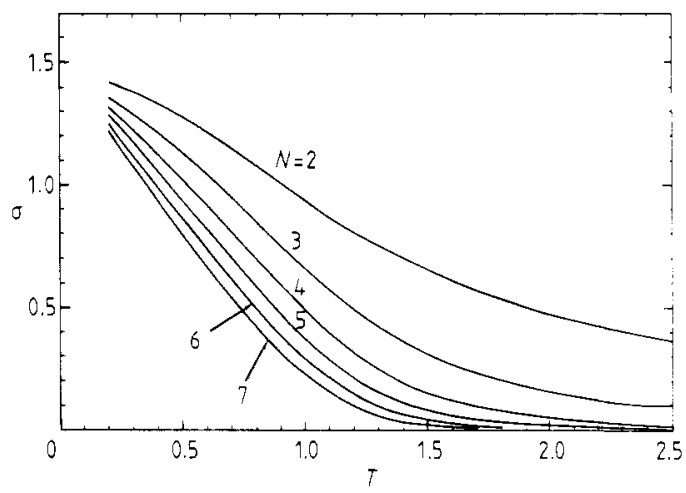

Figure 13. Interface free energy as a function of temperature $(\Delta=0.5)$ for strips of varying widths. The chiral direction is parallel to the strip.

interface properties are sensitive to the paramagnetic-incommensurate transition. We hope in the future to study these quantities further in an attempt to extract accurately the transition temperature and critical properties of this boundary.

\section{Acknowledgments}

JY would like to thank P D Beale, J Chalker and P M Duxbury for helpful conversations and express her appreciation to le Service de Physique Théorique du Saclay for their hospitality while this work was in progress.

\section{References}

Barber M N 1983 in Phase Transitions and Critical Phenomena vol 8, ed C Domb and J Lebowitz (New York: Academic)

Camp W J and Fisher M E 1972 Phys. Rev. B 6946

den Nijs M 1984 J. Phys. A: Math. Gen. 17 L295

Duxbury P M, Yeomans J M and Beale P D 1984 J. Phys. A: Math. Gen. 17 L179

Haldane F D M, Bak P and Bohr T 1983 Phys. Rev. B 282743

Houlrik J M, Knak Jenson S J and Bak P Phys. Rev. B 282883

Howes S F 1982 Phys. Rev. B 271762

Huse D A 1981 Phys. Rev. B 245180

Huse D A, Szpilka A M and Fisher M E 1983 Physica 121A 363

Kinzel W and Yeomans J M 1981 J. Phys. A: Math. Gen. 14 L163

Ostlund S 1981 Phys. Rev. B 24398

Schultz H J 1983 Phys. Rev. B 282746

Selke W 1984 Surface Sci. 144176

Selke W and Huse D A 1983 Z. Phys. B 50113

Selke W and Pesch W 1982 Z. Phys. B 47335

Selke W and Yeomans J M 1982 Z. Phys. B 46311

von Gehlen G and Rittenberg V 1983 Preprint University of Bonn

Wu F Y 1982 Rev. Mod. Phys. 54235 